\title{
Average Rate and Outage Probability of Cyclic Prefixed Single-Carrier Opportunistic Cooperative Diversity Systems
}

\author{
Kyeong Jin Kim*, Theodoros A. Tsiftsis ${ }^{\dagger}$, and George K. Karagiannidis ${ }^{\ddagger}$ \\ *9800 Sunrise Court, Irving, TX 75063, E-mail: kyeong.j.kim@hotmail.com. \\ $\dagger^{\dagger}$ Department of Electrical Engineering, Technological Educational Institute (TEI) of Lamia, 35100 Lamia, Greece, Email: tsiftsis@teilam.gr.

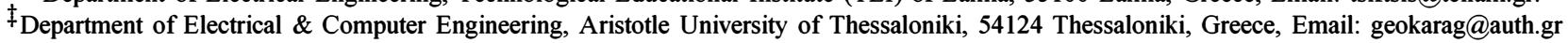

\begin{abstract}
In this paper, several performance analysis metrics for the cyclic prefix-based single-carrier (CP-SC) opportunistic cooperative diversity systems are presented. After the statistical evaluation of the end-to-end signal-to-noise-ratio of a two-hop relaying transmission, we derive tight upper bounds for the maximum achievable average rate and lower bounds for the outage probability in closed form. Further, asymptotic analysis on the outage probability reveals that the diversity gain is determined by both the number of relay nodes in the system and the number of channel taps being supported by the CP length. Simulation results verify the derived closed-form analytical expressions and also the diversity gains. Asymptotic performance is also verified via Monte Carlo simulations.
\end{abstract}

\section{INTRODUCTION}

Orthogonal frequency division multiplexing (OFDM) has been so popular in several wireless systems [1], [2]. However, it has several inherent problems such as large peak-to-average ratio, large power-backing off, and the need of linear amplifiers having large dynamic range [3]. To deal with these problems, the cyclic prefix-based single-carrier (CP-SC) transmission [4] has started to be adopted in the wireless systems such as the mmWave Wireless Personal Area Networks (WPAN) targeting in-flight entertainment distribution and the wireless version of the High-Definition Multimedia Interface (HDMI), gaming interfaces, and high-speed backhaul and content distribution services [5], [6].

Relay networks become more attractive in the LTEAdvanced [7] and WiMAX [2] standard development. There are few approaches using CP-SC in the relay transmission [8], [9]. In [8], [9], the diversity gain is analyzed for the block coded SC system. In the relaying transmission, there are several relay protocols such as amplify-and-forward (AF), decode-and-forward (DF), and coded cooperation using two hops or several hops [10].

In this paper, opportunistic relaying can be implemented in a CP-SC cooperative diversity system (CDS) either with the appropriate selection cooperation algorithm where the "best" relay node is selected in the destination terminal [11], [12] or in a distributed manner among relays via opportunistic relaying [13]. We first define the equivalent instantaneous endto-end signal-to-noise ratio (SNR) of a two hop transmission in a CP-SC CDS using the properties of circulant channel matrices. Due to the mathematical complexity of the derived SNR, we use a very well known upper bound [14] and then we investigate its statical behavior. Based on this upper bound for the end-to-end SNR, we are able to derive tight upper bounds for the maximum achievable average rate and tight lower bounds for the outage probability in closed form. To find the diversity gain of the CP-SC CDS with opportunistic relaying, the outage probability is also analyzed in practical high SNR regions.

From asymptotic performance analysis, it is mathematically proved and shown in the presented graphs that the diversity gain is determined by both the number of relay nodes in the system and the number of multipath signals. That is, the multiuser diversity gain and the multipath diversity gain simultaneously determine the whole diversity gain of the presented CP-SC CDS with "best" relay selection.

Notation: The superscripts ${ }^{*},{ }^{T},{ }^{H}$ stand for complex conjugate, transposition, and conjugate transposition, respectively. $E\{\cdot\}$ denotes statistical expectation; $\boldsymbol{I}_{N}$ is the $N \times N$ identity matrix; 0 stands for an all zeros matrix of appropriate dimensions; $\|\boldsymbol{x}\|$ denotes the norm of the vector $\boldsymbol{x}$, i.e., $\|\boldsymbol{x}\|=\boldsymbol{x}^{H} \boldsymbol{x}$; $\mathcal{N}\left(\mu, \sigma^{2}\right)$ denotes Gaussian distribution of random variable with mean $\mu$ and variance $\sigma^{2}$; and the $(i, j)$-th element of the matrix $\boldsymbol{A}$ is denoted by $(\boldsymbol{A})_{i, j}$.

\section{System AND ChANNEL MOdel}

The proposed relaying system has one single source node (S), which communicates with the destination node (D) via $K$ AF relay nodes. The $i$-th relay node is denoted by $R_{i}$. The CP-SC is used as a transmission technique, whereas a twohop opportunistic relaying without a direct path between the source and the destination node is used as a relay protocol in two time slots. When the "best" relay node is selected via opportunistic relaying [13], the source node sends out its transmission symbol block $\boldsymbol{d}(2 n)$ to the "best" relay node. In the second time slot ${ }^{1}$, the "best" relay amplifies and forwards its data from the source. One transmission block forms $N$ symbols, that is, $\boldsymbol{d}(2 n)=\left[d_{1}(2 n), . ., d_{N}(2 n)\right]^{T}$, of which its

\footnotetext{
${ }^{1}$ Without loss of generality, we assume that the data-sharing phase is implemented in even transmission intervals whereas the relaying phase in odd transmission intervals.
} 
$k$-th symbol element is denoted by $d_{k}(n)$ having property of $E\left\{d_{k}(n) d_{k^{\prime}}(m)^{*}\right\}=E_{s} \delta\left(k-k^{\prime}\right) \delta(n-m)$, where $\delta(\cdot)$ denotes the discrete-time Dirac delta function and $E_{s}=1$. A CP of $N_{g}$ symbols is appended to the front of the transmission block to prevent inter-block-symbol interference (IBSI).

An instantaneous channel between the source and the $i$-th relay is characterized by a channel vector consisting of a set of channel impulse responses $\boldsymbol{h}^{S-R_{i}}(2 n) \triangleq\left[h_{0}^{S-R_{i}}(2 n), . ., h_{N_{f}-1}^{S-R_{i}}(2 n)\right]^{T}$ with $N_{f}$ being the channel order. The received vector signal at the relay node $R_{i}$ after the CP has been eliminated becomes

$$
\tilde{\boldsymbol{y}}^{S-R_{i}}(2 n)=\sqrt{P_{s}} \boldsymbol{H}_{c i r}^{S-R_{i}}(2 n) \boldsymbol{d}(2 n)+\tilde{\boldsymbol{z}}^{S-R_{i}}(2 n),
$$

where $P_{s}$ is the average signal power transmitted by the source node and $\boldsymbol{H}_{\text {cir }}^{S-R_{i}}(2 n) \in \mathbb{C}^{N \times N}$ is a time variant circulant matrix with $\left(\boldsymbol{H}_{c i r}^{S-R_{i}}(2 n)\right)_{j, l}=h_{<j-l>N}^{S-R_{i}}(2 n)$, where $<\cdot>_{N}$ denotes modulo- $N$ operation. In (1), it is assumed that $\tilde{\boldsymbol{z}}^{S-R_{i}}(2 n) \sim \mathcal{C N}\left(\mathbf{0}, \sigma_{n}^{2} \boldsymbol{I}_{N}\right)$ with one-sided power spectral density $N_{0}$. After applying a relay gain matrix $g_{i} \boldsymbol{I}_{N}$ at relay $R_{i}$, the received vector signal at the destination node becomes

$$
\begin{aligned}
& \tilde{\boldsymbol{y}}^{R_{i}-D}(2 n+1)=g_{i} \sqrt{P_{s}} \boldsymbol{H}_{c i r}^{R_{i}-D}(2 n+1) \boldsymbol{H}_{c i r}^{S-R_{i}}(2 n) \boldsymbol{d}(2 n) \\
& +g_{i} \boldsymbol{H}_{c i r}^{R_{i}-D}(2 n+1) \tilde{\boldsymbol{z}}^{S-R_{i}}(2 n)+\tilde{\boldsymbol{z}}^{R_{i}-D}(2 n+1) .
\end{aligned}
$$

In the sequel, we use the following properties of the circulant matrix related operations.

- P1 : Let $\boldsymbol{H}_{c i r}^{S-R i} \in \mathbb{C}^{N \times N}$ and $\boldsymbol{H}_{c i r}^{R_{i}-D} \in \mathbb{C}^{N \times N}$ be exactly known circulant matrices, then $\left(\boldsymbol{H}_{c i r}^{S-R i}\right)^{H}$ and $\boldsymbol{H}_{\text {cir }}^{S-R i} \boldsymbol{H}_{c i r}^{R_{i}-D}$ become circulant matrices [15].

- P2: The orthogonal eigendecomposition of $\boldsymbol{H}_{c i r}^{S-R i}$ is given by

$$
\boldsymbol{H}_{c i r}^{S-R i}=\boldsymbol{W}^{H} \boldsymbol{\Lambda}^{S-R_{i}} \boldsymbol{W},
$$

where $\boldsymbol{W} \in \mathbb{C}^{N \times N}$ is the discrete Fourier transform (DFT) and the diagonal matrix $\Lambda^{S-R_{i}}$ contains the DFT of the first row of $\boldsymbol{H}_{c i r}^{S-R_{i}}$ [16], i.e.,

$$
\lambda_{n}^{S-R_{i}}=\sum_{l=0}^{N_{f}-1} h_{l}^{S-R_{i}} e^{-j 2 \pi n l / N}, n=0, . N-1 .
$$

- P3: With $\boldsymbol{d}(2 n)$ which has the statistical property of $E\left\{\|\boldsymbol{d}(2 n)\|^{2}\right\}=\boldsymbol{I}_{N}$, we have

$$
\begin{aligned}
E\left\{\left\|\boldsymbol{H}_{\text {cir }}^{S-R_{i}} \boldsymbol{d}(2 n)\right\|^{2}\right\} & =\operatorname{trace}\left(\left(\boldsymbol{H}_{\text {cir }}^{S-R_{i}}\right)^{H} \boldsymbol{H}_{\text {cir }}^{S-R_{i}}\right) \\
& =\sum_{m=0}^{N-1} \tilde{\lambda}_{m}^{S-R_{i}}
\end{aligned}
$$

where $\tilde{\lambda}_{m}^{S-R_{i}} \triangleq \sum_{l, l^{\prime}=0}^{N_{f}-1} h_{l}^{S-R_{i}}\left(h_{l^{\prime}}^{S-R_{i}}\right)^{*} e^{-j 2 \pi m\left(l-l^{\prime}\right) / N}$. - P4: With available $\boldsymbol{H}_{\text {cir }}^{S-R_{i}}$ and $\boldsymbol{H}_{c i r}^{R_{i}-D}$, it is given by

$$
\begin{aligned}
E & \left\{\left\|\boldsymbol{H}_{c i r}^{S-R_{i}} \boldsymbol{H}_{c i r}^{R_{i}-D} \boldsymbol{d}(2 n)\right\|^{2}\right\} \\
& =\operatorname{trace}\left(\left(\boldsymbol{H}_{c i r}^{R_{i}-D}\right)^{H}\left(\boldsymbol{H}_{c i r}^{S-R_{i}}\right)^{H} \boldsymbol{H}_{c i r}^{S-R_{i}} \boldsymbol{H}_{c i r}^{R_{i}-D}\right) \\
& =\sum_{m=0}^{N-1} \tilde{\lambda}_{m}^{S-R_{i}} \tilde{\lambda}_{m}^{R_{i}-D}
\end{aligned}
$$

where $\tilde{\lambda}_{m}^{R_{i}-D \triangleq} \sum_{l, l^{\prime}=0}^{N_{f}-1} h_{l}^{R_{i}-D}\left(h_{l^{\prime}}^{R_{i}-D}\right)^{*} e^{-j 2 \pi m\left(l-l^{\prime}\right) / N}$.

\section{INSTANTANEOUS END-TO-END SNR}

The equivalent instantaneous end-to-end SNR between the source and the destination node via the $i$-th relay node is given by [17]

$$
\begin{aligned}
\gamma_{i} & \triangleq \frac{S_{i}}{N_{i}} \\
& =\frac{\frac{1}{N} P_{s} E\left\{\left\|g_{i} \boldsymbol{H}_{c i r}^{R_{i}-D}(2 n+1) \boldsymbol{H}_{c i r}^{S-R_{i}}(2 n) \boldsymbol{d}(2 n)\right\|^{2}\right\}}{\frac{1}{N} E\left\{\left\|g_{i} \boldsymbol{H}_{c i r}^{R_{i}-D}(2 n+1) \tilde{\boldsymbol{z}}^{S-R_{i}}(2 n)+\tilde{\boldsymbol{z}}^{R_{i}-D}(2 n+1)\right\|^{2}\right\}}
\end{aligned}
$$

Using P3 and P4, (7) can be expressed alternatively

$$
\gamma_{i}=\frac{\frac{P_{s} g_{i}^{2}}{N} \sum_{m=0}^{N-1} \tilde{\lambda}_{m}^{S-R i} \tilde{\lambda}_{m}^{R i-D}}{\frac{g_{i}^{2}}{N} \sum_{m=0}^{N-1} \tilde{\lambda}_{m}^{R i-D} \sigma_{n}^{2}+\sigma_{n}^{2}} .
$$

The $i$-th relay gain is obtained from [18]; this gain aims at limiting the "best" relay's output power,

$$
g_{i}=\sqrt{\frac{P_{r}}{P_{s} \frac{1}{N} \sum_{m=0}^{N-1} \tilde{\lambda}_{m}^{S-R_{i}}+\sigma_{n}^{2}}} .
$$

By substituting (9) into (8), yields

$$
\gamma_{i}=\frac{\frac{P_{s}}{\sigma_{n}^{2}} \frac{P_{r}}{\sigma_{n}^{2}} \frac{1}{N} \sum_{m=0}^{N-1} \tilde{\lambda}_{m}^{R_{i}-D} \tilde{\lambda}_{m}^{S-R_{i}}}{\left(\frac{P_{s}}{\sigma_{n}^{2}} \frac{1}{N} \sum_{m=0}^{N-1} \tilde{\lambda}_{m}^{S-R_{i}}+\frac{P_{r}}{\sigma_{n}^{2}} \frac{1}{N} \sum_{m=0}^{N-1} \tilde{\lambda}_{m}^{R_{i}-D}+1\right)}
$$

\section{A. Distribution of the Upper Bound of the Average End-to- End SNR}

If the elements of $\boldsymbol{h}^{S-R_{i}}(2 n)$ and $\boldsymbol{h}^{R_{i}-D}(2 n+1)$ are independent and identically distributed (i.i.d.) processes, then the average end-to-end signal power and the average endto-end noise power over channel vectors $\boldsymbol{h}^{S-R_{i}}(2 n)$ and $\boldsymbol{h}^{R_{i}-D}(2 n+1)$ are given by, respectively,

$$
\begin{aligned}
\tilde{S}_{i} \triangleq & \frac{P_{s}}{\sigma_{n}^{2}} \frac{P_{r}}{\sigma_{n}^{2}} E\left\{\frac{1}{N} \sum_{m=0}^{N-1} \tilde{\lambda}_{m}^{R_{i}-D} \tilde{\lambda}_{m}^{S-R_{i}}\right\} \\
= & \gamma^{S-R_{i}} \gamma^{R_{i}-D}, \\
\tilde{N}_{i} \triangleq & \frac{P_{s}}{\sigma_{n}^{2}} E\left\{\frac{1}{N} \sum_{m=0}^{N-1} \tilde{\lambda}_{m}^{S-R_{i}}\right\} \\
& +\frac{P_{r}}{\sigma_{n}^{2}} E\left\{\frac{1}{N} \sum_{m=0}^{N-1} \tilde{\lambda}_{m}^{R_{i}-D}\right\}+1 \\
= & \gamma^{S-R_{i}}+\gamma^{R_{i}-D}+1,
\end{aligned}
$$

where $\quad \gamma^{S-R_{i}} \triangleq P_{s} \sum_{k=0}^{N_{f}-1}\left|h_{k}^{S-R_{i}}(2 n)\right|^{2} / \sigma_{n}^{2} \quad$ and $\gamma^{R_{i}-D} \triangleq P_{r} \sum_{l=0}^{N_{f}-1}\left|h_{l}^{R_{i}-D}(2 n+1)\right|^{2} / \sigma_{n}^{2}$. Using (11) and (12), the average end-to-end SNR $\tilde{\gamma}_{i}$ becomes a random variable (RV) w.r.t. $\gamma^{S-R_{i}}$ and $\gamma^{R_{i}-D}$ when the channel impulse responses are i.i.d. RVs, that is,

$$
\tilde{\gamma}_{i} \triangleq \frac{\tilde{S}_{i}}{\tilde{N}_{i}}=\frac{\gamma^{S-R_{i}} \gamma^{R_{i}-D}}{\gamma^{S-R_{i}}+\gamma^{R_{i}-D}+1} .
$$


Theorem 1: The average end-to-end SNR, $\tilde{\gamma}_{i}$, is upper bounded by

$$
\begin{aligned}
\tilde{\gamma}_{i} & =\frac{\gamma^{R_{i}-D} \gamma^{S-R_{i}}}{\left(\gamma^{R_{i}-D}+\gamma^{R_{i}-D}+1\right)} \leq \min \left(\gamma^{S-R_{i}}, \gamma^{R_{i}-D}\right) \\
& =\frac{P_{T}}{2 \sigma_{n}^{2}} \min \left(\tilde{\gamma}^{S-R_{i}}, \tilde{\gamma}^{R_{i}-D}\right)=a \gamma_{i}^{u p}
\end{aligned}
$$

where it is assumed the total transmission power $P_{T}$ is equally allocated to the source and the selected relay i.e., $P_{s}=P_{r}=\frac{P_{T}}{2}, a \triangleq \frac{P_{T}}{2 \sigma_{n}^{2}}, \tilde{\gamma}^{S-R_{i}} \triangleq \sum_{k=0}^{N_{f}-1}\left|h_{k}^{S-R_{i}}(2 n)\right|^{2}$, and $\tilde{\gamma}^{R_{i}-D} \triangleq \sum_{l=0}^{N_{f}-1}\left|h_{l}^{R_{i}-D}(2 n+1)\right|^{2}$. When the channel impulse responses are distributed as i.i.d. complex Gaussian RVs with zero mean and unit variance $\mathcal{C N}(0,1)$, that is, $h_{k}^{S-R_{i}}(2 n) \sim \mathcal{C N}(0,1)$ and $h_{k}^{R_{i}-D}(2 n+1) \sim \mathcal{C N}(0,1)$, for $k=0, . ., N_{f}-1$, then the probability density function (PDF) and the cumulative distribution function (CDF) of $\gamma_{i}^{u p}$ are, respectively, given by

$$
\begin{aligned}
& f_{\gamma_{i}^{u p}}(x)=\frac{2}{\left(N_{f}-1\right) !}(x)^{N_{f}-1} e^{-2 x} \sum_{l=0}^{N_{f}-1} \frac{1}{l !}(x)^{l} u(x<15) \\
& F_{\gamma_{i}^{u p}}(x)=1-\left(e^{-x} \sum_{l=0}^{N_{f}-1} \frac{x^{l}}{l !}\right)^{2}
\end{aligned}
$$

where $u(x)$ denotes the unit step function.

Proof: We can readily show that $\tilde{\gamma}^{S-R_{i}}$ and $\tilde{\gamma}^{R_{i}-D}$ have chi-squared distributions with $2 N_{f}$ degrees of freedom, of which their PDFs are given by

$\tilde{\gamma}^{R_{i}-D}=\tilde{\gamma}^{S-R_{i}}=\tilde{\gamma} \sim f_{\tilde{\gamma}}(x)=\frac{1}{\left(N_{f}-1\right) !} x^{N_{f}-1} e^{-x} u(x)$.

The corresponding CDF is given by

$$
F_{\tilde{\gamma}}(x) \triangleq \operatorname{Pr}(\tilde{\gamma} \leq x)=1-e^{-x} \sum_{l=0}^{N_{f}-1} \frac{1}{l !} x^{l} .
$$

Using (17), (18), and [19, eqs. (6-56) and (6-58)], we obtain

$$
\begin{aligned}
& f_{\gamma_{i}^{u p}}(x)=\frac{2}{\left(N_{f}-1\right) !}(x)^{N_{f}-1} e^{-2 x} \sum_{l=0}^{N_{f}-1} \frac{1}{l !} x^{l} u(x)(19) \\
& F_{\gamma_{i}^{u p}}(x)=1-\left(e^{-x} \sum_{l=0}^{N_{f}-1} \frac{x^{l}}{l !}\right)^{2}
\end{aligned}
$$

Note that in the derivation of (14), we obtain $g_{i}$ taking into account the statistical properties of the channels [20], [21]. Theorem 1 is useful in computing the PDF and the CDF for $a \gamma_{i}^{u p}$. In deriving (20), all channel elements are assumed to be independent of the relay and transmission time indices. Note also that we can also use the harmonic mean [22]. However, the outage probability expression would be much complicated and thus we were not able to derive exact closed-form expressions both for the average rate and the average symbol error rate of the CP-SC CDS with "best" relay selection. Therefore, we use upper bounds which lead us to gain much insight on the derived diversity gains. Without loss of generality, we will only focus on the case of the same number of channel impulse responses in the relaying system. However, it can be generalized easily extended to the case of the different number of channel impulse responses.

\section{Performance Analysis of the CP-SC CDS With OPPORTUNISTIC RELAYING}

In this section, we will analyze the performance of the CPSC CDS with "best" relay selection. The closed-expressions for the average throughput, and the outage probability will be derived based on (14).

\section{A. Average Throughput}

Since each relay node experiences a different channel characteristics in the time variant environment, we can improve the achievable rate of the CP-SC cooperative diversity by opportunistic relaying.

In a CP-SC CDS system, the maximum achievable average rate is given by

$$
R_{N_{f}, K}=\frac{1}{2} \int_{0}^{\infty} \log _{2}(1+x) f_{\gamma_{\max }}(x) d x
$$

where $\gamma_{\max } \triangleq a \max \left\{\gamma_{1}^{u p}, . ., \gamma_{K}^{u p}\right\}$ is a random variable with $f_{\gamma_{\max }}(x)$, which is computed as follows:

$$
\begin{aligned}
f_{\gamma_{\max }}(x)= & K\left(F_{\gamma_{i}^{a p p, u p}}\left(\frac{x}{a}\right)\right)^{K-1}\left(\frac{1}{a} f_{\gamma_{i}^{a p p, u p}}\left(\frac{x}{a}\right)\right) \\
= & \frac{2 K}{a\left(N_{f}-1\right) !} \sum_{k=0}^{K-1}\left(\begin{array}{c}
K-1 \\
k
\end{array}\right)(-1)^{k} e^{-\frac{2 x}{a}(k+1)} \\
& \times\left(\frac{x}{a}\right)^{N_{f}-1}\left(\sum_{l=0}^{N_{f}-1} \frac{1}{l !}\left(\frac{x}{a}\right)^{l}\right)^{2 k+1}
\end{aligned}
$$

Based on the derived $f_{\gamma_{\max }}(x)$, Theorem 2 provides the maximum achievable rate of the CP-SC CDS with selection cooperation.

Theorem 2: The maximum average achievable rate can be evaluated in terms of Meijer G-function as

$$
\begin{aligned}
R_{N_{f}, K}= & \frac{K}{\ln (2) a^{N_{f}}\left(N_{f}-1\right) !} \sum_{k=0}^{K-1}\left(\begin{array}{c}
K-1 \\
k
\end{array}\right)(-1)^{k} \\
& \sum_{l_{1}, l_{2}, \ldots, l_{N_{f}}}^{2 k+1}\left(\frac{(2 k+1) !}{l_{1} ! l_{2} ! \ldots l_{N_{f}} !}\right) \frac{1}{\prod_{t=0}^{N_{f}-1}\left(t ! a^{t}\right)^{l_{t+1}}} \\
& G_{2,3}^{3,1}\left(b(k) \mid \begin{array}{c}
-m, 1-m \\
0,-m,-m
\end{array}\right) .
\end{aligned}
$$

Proof: By using the multinomial theorem in (22) and after 
simpler algebraic manipulations, we obtain

$$
\begin{aligned}
& f_{\gamma_{\max }}(x)=\frac{2 K}{a^{N_{f}}\left(N_{f}-1\right) !} \sum_{k=0}^{K-1}\left(\begin{array}{c}
K-1 \\
k
\end{array}\right)(-1)^{k} \\
& \sum_{l_{1}, l_{2}, \ldots, l_{N_{f}}}^{2 k+1}\left(\frac{(2 k+1) !}{l_{1} ! l_{2} ! \ldots l_{N_{f}} !}\right) \frac{1}{\prod_{t=0}^{N_{f}-1}\left(t ! a^{t}\right)^{l_{t+1}}} x^{m-1} e^{-b(k) x},
\end{aligned}
$$

where $b(k) \triangleq \frac{2(k+1)}{a}, m \triangleq N_{f}+\left(\sum_{t=0}^{N_{f}-1} t l_{t+1}\right)$ and the nonnegative integers $\left[l_{1}, l_{2}, \ldots, l_{N_{f}}\right]$ are such that $\sum_{t=1}^{N_{f}} l_{t}=$ $2 k+1$.

By substituting (24) into (21) we have

$$
\begin{aligned}
R_{N_{f}, K}= & \frac{K}{\ln (2) a^{N_{f}\left(N_{f}-1\right) !}} \sum_{k=0}^{K-1}\left(\begin{array}{c}
K-1 \\
k
\end{array}\right)(-1)^{k} \\
& \sum_{l_{1}, l_{2}, \ldots, l_{N_{f}}}^{2 k+1}\left(\frac{(2 k+1) !}{l_{1} ! l_{2} ! \ldots l_{N_{f}} !}\right) \frac{1}{\prod_{t=0}^{N_{f}-1}\left(t ! a^{t}\right)^{l_{t+1}}} \\
& \int_{0}^{\infty} \ln (1+x) x^{m-1} e^{-b(k) x} d x .
\end{aligned}
$$

In order to solve the integral in (25), we express the $\ln (\cdot)$ and the exponential terms of the integrand in terms of the Meijer G-function according to [23, eq. (11)]. Then, (25) can be easily derived by using [23, eq. (21)].

\section{B. Outage Probability}

The end-to-end outage probability at the output of the destination node with maximum output SNR, $P_{\text {out }}$, is defined as $\gamma_{\max }$ falls below a given threshold, $\gamma_{t h}$. Under this condition, the outage probability can be written as

$$
P_{\text {out }}\left(\gamma_{t h}\right) \triangleq \operatorname{Pr}\left[\gamma_{\max } \leq \gamma_{t h}\right]=F_{\gamma_{\max }}\left(\gamma_{t h}\right) .
$$

Using (20), (26) can be written as

$$
P_{\text {out }}\left(\gamma_{t h}\right)=\left(1-\left(e^{-\left(\frac{\gamma_{t h}}{a}\right)} \sum_{l=0}^{N_{f}-1} \frac{\left(\frac{\gamma_{t h}}{a}\right)^{l}}{l !}\right)^{2}\right)^{K}
$$

from which we can find that $N_{f}$ and $K$ are important parameters that determine $P_{\text {out }}\left(\gamma_{t h}\right)$ compared to previous approaches [22], [24]-[26]. In general, $P_{\text {out }}\left(\gamma_{t h}\right)$ approaches to one as either $N_{f}$ or $K$ increases.

\section{Outage Probability at High Average SNR}

Proposition 1: In the high SNR regime (i.e. for sufficiently high $a$ such that $\left.\gamma_{t h} / a \rightarrow 0\right)$, (27) is given by

$$
P_{\text {out }}\left(\gamma_{t h}\right) \approx\left(\frac{2}{N_{f} !}\right)^{K}\left(\frac{\gamma_{t h}}{a}\right)^{K N_{f}}
$$

Proof: The CDF of (27) can be rewritten as

$$
P_{\text {out }}\left(\gamma_{t h}\right)=\left[1-\left(e^{-\frac{\gamma_{t h}}{a}} \sum_{l=0}^{N_{f}-1} \frac{\gamma_{t h}^{l}}{a^{l} l !}\right)^{2}\right]^{K} .
$$

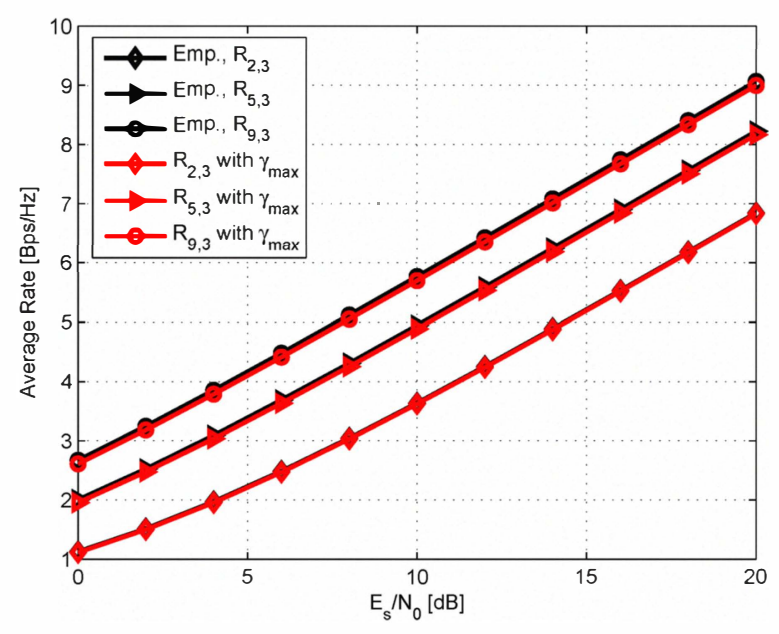

Fig. 1. Maximum average rate at $K=3$ and $N_{f}=\{2,5,9\}$.

By using [27, Eqs. (8.352.2) and (8.354.2)] for the incomplete gamma function $\frac{\Gamma\left[N_{f}-1, \frac{\gamma_{t h}}{a}\right]}{\left(N_{f}-1\right) !}$ yields $^{2}$

$$
\begin{aligned}
& P_{\text {out }}\left(\gamma_{t h}\right)=\left[1-\left(\frac{\Gamma\left[N_{f}, \frac{\gamma_{t h}}{a}\right]}{\left(N_{f}-1\right) !}\right)^{2}\right]^{K} \\
& =\left[1-\left(\frac{\left(N_{f}-1\right) !-\left(\frac{\gamma_{t h}}{a}\right)^{N_{f}} \frac{1}{N_{f}}\left(\left(1+O\left(\frac{\gamma_{t h}}{a}\right)\right)\right.}{\left(N_{f}-1\right) !}\right)^{2}\right]^{K}
\end{aligned}
$$

By selecting only the first order terms in (30), (28) can be easily obtained.

From Proposition 5, it is obvious that the outage diversity gain is $K N_{f}$.

\section{Simulation Results}

In the simulations, we have used $N=512$ for the symbol block size and the quadrature phase-shift keying (QPSK) modulation for data symbols with $P_{T}=1$.

\section{A. Effect of Number of Channel Lengths}

Fig. 1 shows the maximum achievable rate with a different channel length specified by $N_{f}$. Three relay nodes are employed in the system, that is, $K=3$. This plot indicates that the better maximum achievable rate can be obtained as $N_{f}$ increases. A bigger multipath diversity gain influences on this rate improvement. The ensemble average for (21) is also curved in this plot. Theoretically obtained rate using $\gamma_{\max }$ is also plotted as a comparison. It is also shown that the derived closed-form expression in (23) is correct. Compared to previous approaches [22], [24]-[26], Fig. 1 suggests that $N_{f}$ also plays an important role in determining the average rate. It is evident from Fig. 1 that the proposed approaches have

\footnotetext{
${ }^{2} f(x)=O(g(x))$ means there exists a non-negative constant $\tau$ such that i.e., $\lim _{x \rightarrow \infty} \frac{f(x)}{g(x)} \leq \tau$.
} 


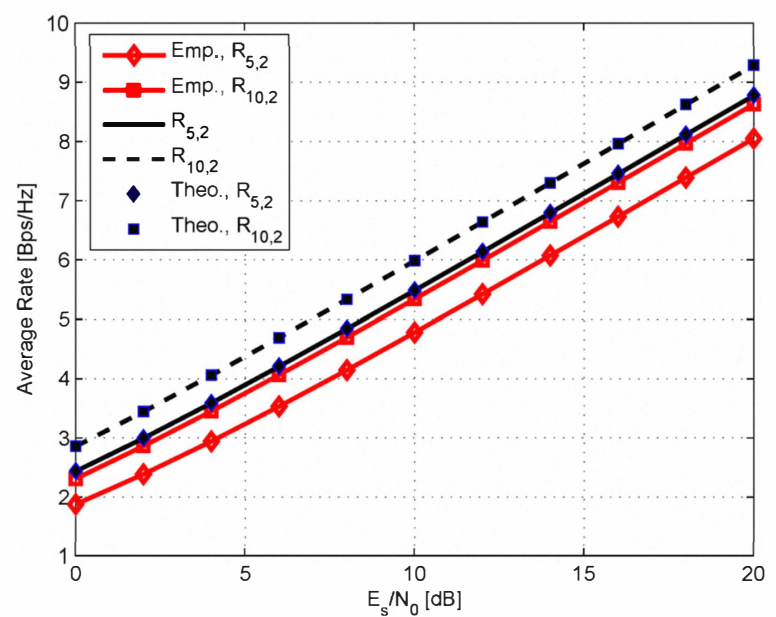

Fig. 2. Maximum average rate at $N_{f}=5$ and $K=\{2,10\}$.

better average rates than the harmonic mean-based approaches [22] since we use the upper bound $\gamma_{i}^{u p}$ defined in (14).

\section{B. Effect of Number of Relay Nodes}

Fig. 2 is the corresponding plot with a different number of relay nodes in the system. As in Fig. 1, the more number of relay nodes $K$, the better maximum achievable rate can be achieved, which shows that a better multiuser diversity gain can be achieved.

\section{Outage Probabilities}

Fig. 3 shows the outage probability $P_{\text {out }}\left(\gamma_{t h}\right)$ as a function of $\left(K, N_{f}\right)$. To obtain $P_{\text {out }}\left(\gamma_{t h}\right)=\alpha, 0<\alpha<1$, a bigger threshold $\gamma_{t h}$ is required as either $N_{f}$ or $K$ increases. To find the asymptotic outage probability diversity gain as a function of $\gamma_{t h} / \alpha$, we use (28), which shows that the asymptotic outage probability diversity gain is determined by the number of relays $K$ and the channel length $N_{f}$ in the system. To verify this diversity gain, we use $\gamma_{t h}=\{0.0313,0.1563,0.3125\}$ in the simulations. From several curves plotted in a log$\log$ scale in Fig. 4, we find the outage probability diversity gain that is independent of $\gamma_{t h}$. For instance, $\left(\gamma_{t h}=\right.$ $0.0313, K=3, N_{f}=3$ ) has the same diversity $G_{d}=9$ as $\left(\gamma_{t h}=0.3125, K=9, N_{f}=1\right)$, while we have diversity gain $G_{d}=12$ from $\left(\gamma_{t h}=0.1563, K=4, N_{f}=3\right)$ and $\left(\gamma_{t h}=0.3125, K=6, N_{f}=2\right)$. From these results, the better outage probability diversity gain at a fixed channel length can be obtained by installing more relay nodes in the system, which corresponds to the results of the conventional approaches [28], [29].

\section{CONCLUSIONS}

In this paper, we have derived in closed form tight bounds for the maximum average achievable rate and the outage probability of the CP-based SC opportunistic cooperative diversity systems. The simulation results verified the derived closedform expressions. Moreover, the outage diversity gain has also

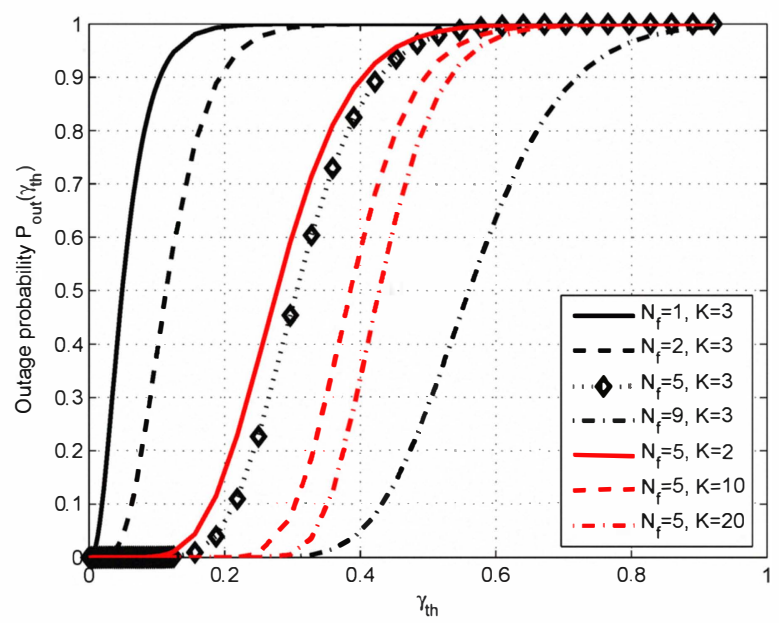

Fig. 3. Outage probability at various number of relays and channel lengths.

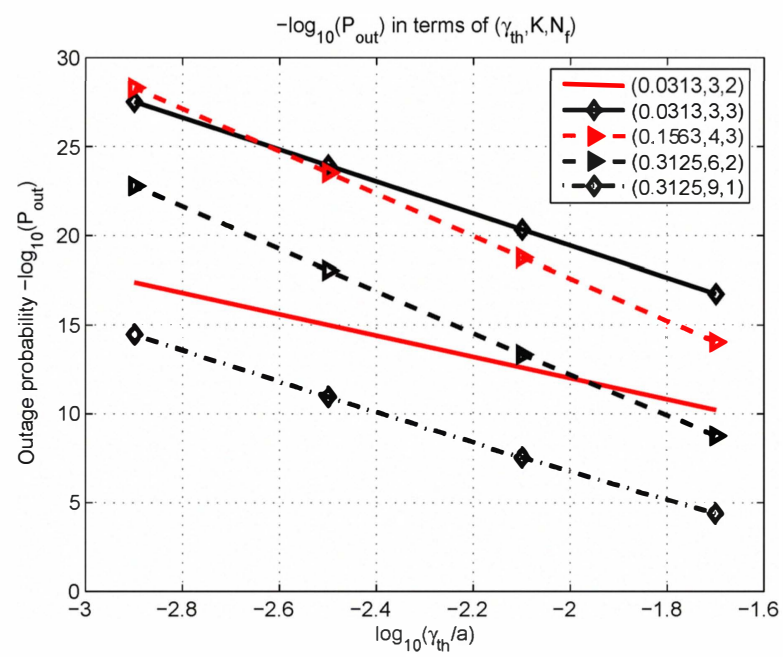

Fig. 4. Asymptotic outage probability showing diversity gain at various number of relays and channel lengths.

been derived. Monte-Carlo simulations verified that the outage diversity gain is determined by the number of the relay nodes and the length of the channel taps, simultaneously.

\section{REFERENCES}

[1] IEEE P802.11n/D1.04, "Wireless LAN medium access control (MAC) and physical layer (PHY) specifications: Enhancements for higher throughput," Sept. 2006.

[2] IEEE 802.16e/D9, "Air interface for fixed and mobile broadband wireless access systems - amendment for physical and medium access control layers for combined fixed and mobile operation in licensed bands," June 2005.

[3] S. Kato, H. Harada, R. Funada, T. Baykas, C. S. Sum, J. Wang, and M. A. Rahman, "Single carrier transmission for multi-gigabit $60-\mathrm{GHz}$ WPAN systems," IEEE Journal on Select. Areas in Commun., vol. 27, pp. 1466-1478, Oct. 2009.

[4] B. Devillers, J. Louveaux, and L. Vandendorpe, "About the diversity in cyclic prefixed single-carrier systems," Elsevier Physical Communication Journal, vol. 1, pp. 266-276, Dec. 2008. 
[5] IEEE P802.15.3c/D00, "Wireless Medium Access Control (MAC) and Physical Layer (PHY) Specifications for High Rate Wireless Personal Area Networks (WPANs): Amendment 2: Millimeter-wave based Alternative Physical Layer Extension," 2008.

[6] P. Smulders, "Exploiting the $60 \mathrm{GHz}$ band for local wireless multimedia access: prospects and future directions," IEEE Commun. Magazine, pp. 140-147, Jan. 2002.

[7] 3GPP, TR 36.913, "Requirements for further advancements fore-utra(lteadvanced)(release8)," 2009.

[8] D.-Y. Seol, U.-K. Kwon, G.-H. Im, and E.-S. Kim, "Relay-based single carrier transmission with SFBC in uplink fast fading channels," IEEE Commun. Lett., vol. 12, pp. 928-930, Dec. 2007.

[9] D.-Y. Seol, U.-K. n Kwon, and G.-H. Im, "Performance of single carrier transmission with cooperative diversity over fast fading channels," IEEE Trans. Commun., vol. 57, pp. 2799-2807, Sept. 2009.

[10] E. Zimmermann, P. Herhold, and G. Fettweis, "On the performance of cooperative relaying protocols in wireless networks," European Transactions on Telecommunications, vol. 16, pp. 5-16, Jan. 2005.

[11] Y. Zhao, R. Adve, and T. J. Lim, "Symbol error rate of selection amplifyand-forward relay systems," vol. 10, no. 11, pp. $757-759$, Nov. 2006.

[12] _ " "Improving amplify-and-forward relay networks: optimal power allocation versus selection," vol. 6, no. 8, pp. 3114 -3123, Aug. 2007.

[13] A. Bletsas, A. Khisti, D. P. Reed, and A. Lippman, "A simple cooperative diversity method based on network path selection," IEEE Journal on Select. Areas in Commun., vol. 24, pp. 659-672, Mar. 2006.

[14] S. Ikki and M. Ahmed, "Performance analysis of cooperative diversity wireless networks over Nakagami-m fading channel," IEEE Commun. Lett., vol. 11, no. 4, pp. 334-336, April 2007.

[15] G. E. Trap, "Inverse of circulant matrices and block circulant matrices," Kyungbook Math. J., vol. 13, pp. 11-20, 1973.

[16] T.-H. Pham, Y.-C. Liang, A. Nallanathan, and H. Garg, "Optimal training sequences for channel estimation in bi-directional relay networks with multiple antennas," IEEE Trans. Commun., vol. 58, pp. $474-479$, Feb. 2010 .

[17] W. Jiang, Y. Li, and X. Yu, "Truncation for low-complexity MIMO signal detection," IEEE Trans. Inf. Theory, vol. IT-53, pp. 1564-1571, April 2007.

[18] J. N. Laneman and G. W. Wornell, "Energy-efficient antenna sharing and relaying for wireless networks," in Proc. IEEE Wireless Communications and Networking Conf. (WCNC'00), Chicago, IL, Oct. 2000, pp. 7-12.

[19] A. Papoulis, Probability, random variables, and stochastic processes. New York, N.Y.: Wiley, 1963.

[20] H. Yomo and E. D. Carvalho, "A CSI estimation method for wireless relay network," IEEE Commun. Lett., vol. 11, pp. 480-482, June 2007.

[21] C. S. Patel, G. L. Stuber, and T. G. Pratt, "Statistical properties of amplify and forward relay fading channels," IEEE Trans. Veh. Technology., vol. 55, pp. 1-9, Jan. 2006

[22] M. Mazen O. Hasna and M.-S. Alouini, "Harmonic mean and endto-end performance of transmission systems with relays," IEEE Trans. Commun., vol. 52, pp. 130-135, Jan. 2004.

[23] V. S. Adamchik and O. I. Marichev, "The algorithm for calculating integrals of hypergeometric type functions and its realization in REDUCE system," in Proc. International Conference on Symbolic and Algebraic Computation, Tokyo, Japan, 1990, pp. 212-224.

[24] A. Bletsas, H. Shin, and M. Z. Win, "Outage analysis for cooperative communication with multiple amplify and forward relays," IEEE Commun. Lett., vol. 11, pp. 353-355, 2007.

[25] G. Li and H. Liu, "On the capacity of the broadband relay networks," in Proc. Asilomar Conf. Signals, Syst., Comput., Pacific Grove, CA, Nov. 2004, pp. 1318-1322.

[26] S. Suchitra and K. Chang, "Closed-form solution of outage capacity for cooperative DF and AF relay network," Wireless Personal Communications, June 2009.

[27] I. S. Gradshteyn and I. M. Ryzhik, Table of integrals, series, and products. New York, N.Y.: Academic Press, 2007.

[28] B. Barua, H. Q. Ngo, and H. Shin, "On the SEP of cooperative diversity with opportunistic relaying," IEEE Commun. Lett., vol. 12, pp. 727-729, Oct. 2008

[29] A. Bletsas, H. Shin, and W. M.Z, "Outage optimality of opportunistic amplify-and-forward relaying," IEEE Commun. Lett., vol. 11, pp. 261263, March 2007. 